Artículo

\title{
Efectos de la intervención en conciencia fonológica y velocidad de denominación sobre el aprendizaje de la escritura
}

\author{
Rosa María González ${ }^{\mathrm{a}, *}$, Fernando Cuetos ${ }^{\mathrm{b}}$, Juan $\operatorname{Vilar}^{\mathrm{c}}$ y Eva Uceira ${ }^{\mathrm{d}}$ \\ a Departamento de Psicología Evolutiva y de la Educación, Campus de Elviña, Universidad de A Coruña, A Coruña, España \\ b Departamento de Psicología, Universidad de Oviedo, Oviedo, Asturias, España \\ ' Departamento de Matemáticas, Campus de Elviña, Universidad de A Coruña, A Coruña, España \\ d ASPANAES-A Coruña, A Coruña, España
}

\section{INFORMACIÓN DEL ARTÍCULO}

\section{Historia del artículo:}

Recibido el 24 de febrero de 2014

Aceptado el 3 de junio de 2014

On-line el 4 de noviembre de 2014

\section{Palabras clave:}

Conciencia fonológica

Velocidad denominación

Escritura

Aprendizaje

Prevención

\section{R E S U M E N}

El objetivo de este estudio fue analizar el efecto que la intervención conciencia fonológica y velocidad de denominación tiene sobre el aprendizaje de la escritura. Los participantes fueron 271 alumnos, 138 del grupo experimental y 133 del control. Los alumnos del grupo experimental recibieron instrucción en conciencia fonológica y en velocidad de denominación a lo largo de 3 cursos y el grupo control siguió el plan curricular oficial. La instrucción se llevó a cabo con los alumnos de $2 \circ$ de infantil, $3 \circ$ de infantil y $1 \circ$ de primaria, a quienes más tarde se les evaluó la escritura con una prueba estandarizada. El grupo experimental obtuvo puntuaciones significativamente más altas que el grupo control en las pruebas de conciencia fonológica y denominación rápida. Y más importante aún, obtuvo mejores puntuaciones en las tareas de escritura. Estos resultados tienen importantes implicaciones teóricas y educativas que apoyan la relación entre conciencia fonológica y velocidad de denominación en el aprendizaje de la escritura y proporcionan pautas concretas para el trabajo en el aula.

(C) 2014 Instituto de Ciencias de la Educación de la Universidad de Oviedo. Publicado por Elsevier España, S.L.U. Todos los derechos reservados.

\section{Effects of the intervention on phonological awareness and naming speed on learning to write}

\begin{abstract}
A B S T R A C T
The aim of this research was to analyze the causal effect that intervention on phonological awareness and naming speed could have on learning to write. A total of 271 students participate (138 experimental group and 133 control group). The experimental group received instructions on phonological awareness and naming speed throughout three courses, and the control group followed the official curriculum. The instructions given to students of pre-school education (4 and 5 years old) and first grade of primary school ( 6 years old), were evaluated in writing with standardized test three years later. The experimental group obtained higher scores than the control group in the phonological and rapid naming tasks. And more interesting, the experimental group obtained better results in writing tasks. These results have important theoretical implications, as they show the relationship between phonological awareness and naming speed in learning to write. There are also educational implications, since it provides guidelines to facilitate the learning of writing in the classroom.
\end{abstract}

(c) 2014 Instituto de Ciencias de la Educación de la Universidad de Oviedo. Published by Elsevier España, S.L.U. All rights reserved.

\footnotetext{
* Autor para correspondencia. Universidad de A Coruña, Departamento de Psicología Evolutiva y de la Educación, Campus de Elviña, s/n, 15071 A Coruña, España. Correo electrónico: romagose@udc.es (R.M. González).
} 


\section{Introducción}

Procesamiento fonológico y la velocidad de denominación son 2 habilidades cognitivas estrechamente asociadas al aprendizaje de la lectura y la escritura. De hecho, son consideradas precursoras importantes de las mismas, ya que junto con el conocimiento del alfabeto y la memoria fonológica tienen una alta correlación con el progreso inicial de la lectura y el deletreo (National Early Literacy Panel, 2008; Thomson y Hogan, 2009).

La conciencia fonológica se refiere al conocimiento de que el habla se puede segmentar en unidades menores. Hay varios niveles de conciencia fonológica dependiendo de cuál sea la unidad de segmentación: conciencia léxica, cuando las unidades son palabras; conciencia silábica, cuando las unidades son sílabas; conciencia intrasilábica, cuando son partes de la sílaba, como es el caso de la rima, y conciencia fonémica, cuando la unidad de segmentación es el fonema. Todos estos niveles de conciencia fonológica son importantes para el aprendizaje de la lectoescritura, pero especialmente es determinante la conciencia fonémica (Treiman, 2004). Aunque también es la más difícil de adquirir, ya que los fonemas son unidades abstractas que generalmente se descubren durante el aprendizaje de la lectoescritura. Lo que implica una doble dirección puesto que el desarrollo de la conciencia fonémica facilita el aprendizaje de la lectoescritura y el aprendizaje de la lectoescritura es decisivo en el desarrollo de la conciencia fonémica. Como resultado de esta bidireccionalidad, los alumnos con mejores resultados en lectura y escritura también obtienen mejores resultados en conciencia fonológica (Wagner, Torgesen y Rashotte, 1994). Especialmente, en los sistemas ortográficos transparentes como el castellano, en los que existe una clara correspondencia grafemafonema, es suficiente un corto período de entrenamiento en el lenguaje escrito para conseguir una conciencia explícita de los fonemas (Defior, 2008; Defior y Serrano, 2011; Hulme et al., 2002).

Relacionada con la conciencia fonológica está la velocidad de denominación, o capacidad de nombrar rápidamente estímulos visuales altamente familiares, tales como objetos, dígitos, letras o colores. La velocidad de denominación también es un predictor del aprendizaje de la lectoescritura (Suarez-Coalla, García-Castro y Cuetos, 2013). La velocidad de denominación determina la rapidez con la que puede ser recuperada una información fonológica almacenada en la memoria a largo plazo y proporciona información sobre el establecimiento y el uso de las representaciones ortográficas de palabras (Manis, Doi y Bhada, 2000; Manis y Freedman, 2001; Wolf et al., 2002). Refleja la rapidez de procesamiento y requiere la integración de procesos visuales de bajo nivel y de procesos cognitivos y lingüísticos de alto nivel (Manis, Seidenberg y Doi, 1999). La ejecución en tareas de velocidad de denominación es altamente predictora de los logros en el aprendizaje de la lectura y de la escritura en las ortografías transparentes; también lo es, aunque en menor grado, en las opacas (De Jong y van der Leij, 2002; Landerl y Wimmer, 2008; Onochie-Quintanilla, Simpson, Caravolas y Defior, 2011; Defior y Serrano, 2011; Parrila, Kirby y McQuarrie, 2004).

La conciencia fonológica es el mejor predictor del aprendizaje de la lectura (Suárez-Coalla et al., 2013), en el sentido de que los niños prelectores que obtienen mejores puntuaciones en tareas fonológicas son los que aprenden antes a leer. Pero es aún mejor predictor del aprendizaje de la escritura (Furnes y Samuelsson, 2011), lo cual es lógico porque para escribir al dictado es necesario segmentar el habla en fonemas y transformar cada uno de esos fonemas en su correspondiente grafema. Esta transformación es consistente en ortografías transparentes (Caravolas, 2004; Landerl y Wimmer, 2008; Romani, Olson y di Betta, 2005; Verhagen, Aarnoutse y van Leeuwe, 2010). Al mismo tiempo, el aprendizaje de la escritura favorece el desarrollo de la conciencia fonológica, de esta manera, cuando los niños aprenden a escribir aumentan su capacidad para reconocer los fonemas de las palabras (Treiman,
1998). Sin embargo, la relación entre conciencia fonológica y escritura no ha sido tan investigada como la lectura en el ámbito escolar.

De la misma forma, mientras que la asociación entre la velocidad de denominación y la lectura ha sido muy estudiada, la relación entre la velocidad de denominación y la escritura ha recibido menos atención (Sunseth y Bowers, 2002). Y debería correlacionar, al menos, con la escritura de palabras de ortografía arbitraria, puesto que la velocidad de denominación es un índice de acceso léxico y la escritura de palabras de ortografía arbitraria (p. ej., lluvia, yate) requieren el acceso a las representaciones ortográficas en el léxico (Kessler y Treiman, 2003). Algunos estudios realizados en inglés (Savage, Pillay y Melidona, 2008; Strattman y Hodson, 2005) apoyan el papel de la velocidad de denominación como predictor de la escritura. Incluso podría existir una moderada relación entre la velocidad de denominación y la escritura de textos, ya que se ha encontrado que la velocidad de denominación de dígitos en los primeros cursos predice la composición escrita en los cursos superiores (Alburquerque, 2012).

Un problema con la mayor parte de los estudios dedicados a comprobar la relación que la conciencia fonológica y la velocidad de denominación tienen sobre el aprendizaje de la lectura y la escritura es que son de tipo correlacional, en el sentido de demostrar que los niños con mayores puntuaciones en esas tareas también consiguen mejores resultados en lectura y escritura. O que los niños prelectores que realizan mejor las tareas de conciencia fonológica y velocidad de denominación son los que aprenden a leer y escribir más pronto. Pero esos estudios no establecen una relación causa-efecto, ya que las correlaciones podrían deberse a una tercera variable responsable de todas esas tareas. La mejor manera de poder comprobar si existe una relación causa-efecto es interviniendo sobre esos predictores (conciencia fonológica y velocidad de denominación) y comprobando si su mejoría supone también una mejora en la lectura y escritura. Si al entrenar a un niño en conciencia fonológica mejora su lectoescritura, se estará estableciendo una relación causal. Se han hecho algunos estudios en este sentido (McCutchen, 2000; Shanahan, 2004; Swanson y Berninger, 1996), pero aún son escasos y, que sepamos, no hay ninguno realizado en escritura en castellano.

El objetivo de este estudio fue analizar el efecto que la intervención en conciencia fonológica y velocidad de denominación tiene sobre el aprendizaje de la escritura. Para ello se compara el aprendizaje de la escritura de 2 muestras de niños de entre 4 y 6 años, uno que recibe intervención en esas actividades y otro que sigue el curso normal de escolaridad. Nuestra hipótesis es que los niños entrenados en conciencia fonológica y velocidad de denominación obtendrán mejor puntuación en tareas de escritura al dictado.

\section{Método}

\section{Diseño}

El diseño es un estudio cuasiexperimental y longitudinal. Los alumnos de la muestra se dividieron en 2 grupos: el grupo experimental, al cual se le aplicó el programa de intervención, y el grupo control, que siguió la programación oficial establecida para el aprendizaje de la lectoescritura. A todos los participantes se les tomaron medidas pretest y postest en conciencia fonológica y velocidad de denominación al terminar cada curso, además de una medida postest en tareas de escritura, al finalizar el estudio. En la tabla 1 se presenta el número de sesiones para los 2 grupos.

\section{Participantes}

Un total de 271 alumnos (134 niños y 137 niñas) participaron en este estudio, distribuidos según se observa en la tabla 2. 
Tabla 1

Número de sesiones de evaluación e intervención según el curso de inicio

\begin{tabular}{|c|c|c|c|c|c|c|}
\hline Curso al iniciar el estudio & Grupo & $\mathrm{N}$ & $\begin{array}{l}\text { Sesiones de } \\
\text { intervención primer } \\
\text { año de trabajo }\end{array}$ & $\begin{array}{l}\text { Sesiones de } \\
\text { intervención segundo } \\
\text { año de trabajo }\end{array}$ & $\begin{array}{l}\text { Sesiones de } \\
\text { intervención tercer } \\
\text { año de trabajo }\end{array}$ & Total sesiones \\
\hline \multirow[t]{2}{*}{ Segundo de infantil } & Exper. & 45 & 18 & 36 & 36 & 90 \\
\hline & Control & 54 & $\begin{array}{l}\text { Progr. } \\
\text { oficial }\end{array}$ & $\begin{array}{l}\text { Progr. } \\
\text { oficial }\end{array}$ & $\begin{array}{l}\text { Progr. } \\
\text { oficial }\end{array}$ & - \\
\hline \multirow[t]{2}{*}{ Tercero de infantil } & Exper & 46 & 18 & 36 & $\begin{array}{l}\text { Progr. } \\
\text { oficial }\end{array}$ & 54 \\
\hline & Control & 36 & $\begin{array}{l}\text { Progr. } \\
\text { oficial }\end{array}$ & $\begin{array}{l}\text { Progr. } \\
\text { oficial }\end{array}$ & $\begin{array}{l}\text { Progr. } \\
\text { oficial }\end{array}$ & - \\
\hline \multirow[t]{2}{*}{ Primero de primaria } & Exper. & 47 & 18 & $\begin{array}{l}\text { Progr. } \\
\text { oficial }\end{array}$ & $\begin{array}{l}\text { Progr. } \\
\text { oficial }\end{array}$ & 18 \\
\hline & Control & 43 & $\begin{array}{l}\text { Progr. } \\
\text { oficial }\end{array}$ & $\begin{array}{l}\text { Progr. } \\
\text { oficial }\end{array}$ & $\begin{array}{l}\text { Progr. } \\
\text { oficial }\end{array}$ & - \\
\hline
\end{tabular}

Estos alumnos pertenecían a 4 centros educativos, 2 públicos y 2 concertados, situados en zonas urbanas y periféricas de 2 provincias españolas. Dos colegios fueron asignados al azar al grupo experimental (138 alumnos) y los otros 2 al grupo control (133 alumnos). Quedando un colegio público y uno concertado en el grupo experimental, y lo mismo en el grupo control. La lengua escrita de los colegios era el castellano.

\section{Instrumentos y materiales}

\section{Para la evaluación}

La inteligencia general fue evaluada con la Batería de Aptitudes Diferenciales y Generales (BADyG) (EI y E1) (Yuste, 2005a y b), un test compuesto de varias pruebas de aplicación grupal que miden factores como la inteligencia general verbal, inteligencia general no verbal, conceptos cuantitativos y numéricos, información, vocabulario, percepción y coordinación y razonamiento con figuras. Ninguno de los alumnos presentaba alteraciones cognitivas, sensoriales o motrices manifiestas, según se pudo constatar a través de la observación, los informes de los profesores y los resultados de la BADyG. Los resultados de la evaluación inicial indicaron que el grupo experimental y el grupo control eran equiparables en inteligencia general y también en las variables sociodemográficas y edad cronológica, tal como se puede ver en la tabla 2.

Tampoco había diferencias entre el grupo experimental y el control en conciencia fonológica, medida a través de la prueba de aplicación individual PECO (Ramos y Cuadrado, 2006). Esta prueba incluye 6 tareas diferentes: identificación de sílabas, identificación de fonemas, adición de sílabas para formar palabras, adición de fonemas para formar palabras, omisión de una sílaba en palabras y omisión de un fonema en palabras. Se asigna un punto por cada respuesta correcta para una puntuación máxima de 30 , que corresponde al número total de ítems. En la tabla 3 se puede ver que no existían diferencias significativas entre los 2 grupos en ninguna de las 6 tareas y en ninguno de los niveles escolares.

Finalmente, los 2 grupos también estaban emparejados respecto a la velocidad en denominación, evaluada a través de una adaptación de la prueba denominada RAN (Wolf y Denckla, 2005). La prueba de aplicación individual consta de 3 tareas: series de colores (colores primarios para los alumnos de $2 .^{\circ}$ de infantil y colores secundarios para los alumnos de $3 .^{\circ}$ de infantil y $1 .^{\circ}$ de primaria), series de dibujos familiares y series de letras (vocales para los alumnos de 4 años y consonantes invariantes para los alumnos de 5 años y de $1 .^{\circ}$ de primaria). Cada serie incluye 36 estímulos, organizados en 4 filas de 9 elementos cada una. En cada tarea, los estímulos deben ser nombrados de izquierda a derecha, lo más rápido posible. Los tiempos utilizados en la denominación de las diferentes categorías se midieron con cronómetro. De todas las tareas y niveles, solo resultó significativa la velocidad de denominación de dibujos en los alumnos de 3 . $^{\circ}$ de infantil, según se puede observar en la tabla 4.

La escritura se evaluó con el PROESC (Cuetos, Ramos y Ruano, 2002). La prueba es de aplicación grupal y evalúa diferentes aspectos de la escritura. Esta evaluación se hace a través de 4 actividades de dictado: 25 sílabas que reflejan las principales estructuras silábicas; 50 palabras ( 25 palabras de ortografía arbitraria y 25 palabras que siguen reglas ortográficas); 25 palabras inventadas (las 15 últimas sujetas a reglas ortográficas). A los alumnos de $1 .^{\circ}$ de primaria se les aplicó el dictado de sílabas y el dictado de seudopalabras. A los alumnos de $2 .^{\circ}$ y $3 .^{\circ}$ de primaria se les aplicó el dictado de sílabas, de palabras de ortografía arbitraria, reglada y el dictado de seudopalabras.

\section{Para la intervención}

Para el grupo experimental, se diseñó el programa que tenía como objetivo trabajar, de manera explícita, la conciencia fonológica y la velocidad de denominación, 2 veces a la semana y en diferente número de sesiones que tenían una duración, según

Tabla 2

Distribución según curso, edad, sexo e índice de inteligencia

\begin{tabular}{|c|c|c|c|c|c|c|c|c|c|}
\hline \multirow[t]{2}{*}{ Curso al iniciar el estudio } & \multirow[t]{2}{*}{ Sexo, número } & \multirow[t]{2}{*}{ Total alumnos } & \multirow[t]{2}{*}{ Grupo, número } & \multirow{2}{*}{$\begin{array}{l}\text { Edad al iniciar } \\
\text { Media (DT) }\end{array}$} & \multirow{2}{*}{$\begin{array}{l}\text { Edad al finalizar } \\
\text { Media (DT) }\end{array}$} & \multicolumn{4}{|c|}{ Índice general de inteligencia al iniciar } \\
\hline & & & & & & Media (DT) & gl & $\mathrm{F}$ & $\mathrm{p}$ \\
\hline \multirow[t]{4}{*}{ Segundo de infantil } & Niños & 99 & Exper. & $4,04(0,14)$ & $6,75(0,42)$ & $68,51(27,60)$ & 1,97 & 0,95 & 0,332 \\
\hline & 58 & & 45 & & & & & & \\
\hline & Niñas & & Control & $3,93(0,32)$ & $6,64(0,46)$ & $74,20(29,97)$ & & & \\
\hline & 41 & & 54 & & & & & & \\
\hline \multirow[t]{4}{*}{ Tercero de infantil } & Niños & 82 & Exper. & $5,05(0,03)$ & $7,67(0,46)$ & $68,41(24,51)$ & 1,80 & 3,09 & 0,082 \\
\hline & 31 & & 46 & & & & & & \\
\hline & Niñas & & Control & $4,99(0,22)$ & $7,52(0,47)$ & $58,08(28,58)$ & & & \\
\hline & 51 & & 36 & & & & & & \\
\hline \multirow[t]{4}{*}{ Primero de primaria } & Niños & 90 & Exper. & $5,97(0,26)$ & $8,62(0,47)$ & $46,85(31,65)$ & 1,88 & 2,70 & 0,104 \\
\hline & 45 & & 47 & & & & & & \\
\hline & Niñas & & Control & $5,94(0,31)$ & $8,56(0,47)$ & $57,27(28,22)$ & & & \\
\hline & 45 & & 43 & & & & & & \\
\hline
\end{tabular}


Tabla 3

Puntuaciones del grupo experimental y el grupo control en las tareas de conciencia fonológica

\begin{tabular}{|c|c|c|c|c|c|c|}
\hline Curso & Tarea & G. experimental Media (DT) & G. control Media (DT) & gl & $\mathrm{F}$ & $\mathrm{p}$ \\
\hline \multirow[t]{6}{*}{ Segundo de infantil } & Identificación de sílabas & $2,11(1,69)$ & $2,54(1,53)$ & \multirow[t]{6}{*}{1,97} & 1,71 & 0,193 \\
\hline & Identificación fonemas & $1,98(1,68)$ & $2,19(1,56)$ & & 0,40 & 0,528 \\
\hline & Adición de sílabas & $0,02(0,14)$ & $0,04(0,27)$ & & 0,10 & 0,745 \\
\hline & Adición de fonemas & $0,02(0,14)$ & $0,00(0,00)$ & & 1,20 & 0,276 \\
\hline & Omisión de sílabas & $0,13(0,66)$ & $0,43(1,09)$ & & 2,47 & 0,119 \\
\hline & Omisión de fonemas & $0,09(0,28)$ & $0,15(0,68)$ & & 0,29 & 0,589 \\
\hline \multirow[t]{6}{*}{ Tercero de infantil } & Identificación de sílabas & $4,26(0,85)$ & $3,78(1,51)$ & \multirow[t]{6}{*}{1,80} & 3,33 & 0,072 \\
\hline & Identificación fonemas & $3,93(1,28)$ & $3,64(1,51)$ & & 0,91 & 0,342 \\
\hline & Adición de sílabas & $0,85(1,67)$ & $0,53(1,38)$ & & 0,85 & 0,357 \\
\hline & Adición de fonemas & $0,74(1,43)$ & $0,47(1,25)$ & & 0,77 & 0,380 \\
\hline & Omisión de sílabas & $2,67(1,53)$ & $2,39(1,88)$ & & 0,56 & 0,453 \\
\hline & Omisión de fonemas & $0,93(1,28)$ & $1,11(1,70)$ & & 0,28 & 0,595 \\
\hline \multirow[t]{6}{*}{ Primero de primaria } & Identificación de sílabas & $4,49(1,14)$ & $4,19(1,22)$ & \multirow[t]{6}{*}{1,88} & 1,48 & 0,226 \\
\hline & Identificación fonemas & $4,55(1,21)$ & $4,72(, 882)$ & & 0,55 & 0,458 \\
\hline & Adición de sílabas & $3,30(1,50)$ & $2,91(1,64)$ & & 1,38 & 0,242 \\
\hline & Adición de fonemas & $2,68(1,65)$ & $2,84(1,55)$ & & 0,21 & 0,646 \\
\hline & Omisión de sílabas & $3,60(1,66)$ & $3,47(1,35)$ & & 0,16 & 0,685 \\
\hline & Omisión de fonemas & $2,47(1,71)$ & $2,74(1,850)$ & & 0,53 & 0,465 \\
\hline
\end{tabular}

el curso, entre 30 y 45 min. En cada sesión, el protagonista del programa guiaba las actividades a través de diversos materiales manipulativos y multisensoriales que iban secuenciadas de menor a mayor dificultad y que estaban adaptadas a cada edad. La conciencia fonológica se trabajó con tareas de rima, identificación, adición y omisión de sílabas y fonemas, a través de las actividades especialmente lúdicas. Es decir, que los objetivos del programa eran básicamente los mismos para los 3 cursos pero iban en diferente orden, dependiendo del curso. Las actividades ( 3 por objetivo) eran diferentes y se iban complejizando (véase, por ejemplo, en la tabla 5). Las palabras utilizadas fueron seleccionadas de diccionarios de frecuencias (Guerrero y López, 1992; Justicia, 1995). Para intervenir en la velocidad de denominación, se diseñó un panel con series de dibujos, colores o letras que los alumnos, por turnos, debían de nombrar lo más rápido posible y de manera correcta, registrándose los tiempos de ejecución; de tal manera que semanalmente cada alumno leía una serie. Se diseñó también, un cuaderno que permitiera a los alumnos desarrollar aquellas actividades de cada objetivo que requerían lápiz y papel, y que les permitían acceder a los dibujos y a las palabras sobre las que había que desarrollar la actividad pertinente.

El programa está planteado por niveles de tal manera que los niños de $1 .^{\circ}$ de primaria hicieron 18 sesiones en un curso y los de $2 .^{\circ}$ de infantil 90 sesiones en 3 cursos. Se trata de un único programa que tiene una secuencia lógica de dificultad según la edad. En todas las sesiones, se trabajaron la conciencia fonológica y la velocidad de denominación.

Como se muestra en la tabla 5 , los objetivos fueron los mismos o similares y los contenidos se fueron complejizando a medida que avanzaba el curso, únicamente cambiaban de orden y la dificultad de los mismos según la edad.

El grupo control (que pertenecía a colegios diferentes del grupo experimental) siguió la enseñanza reglada. Se pasó un cuestionario a los profesores al inicio del trabajo para que describieran los contenidos y la metodología de enseñanza de la lectoescritura que tenían programada, y al final del estudio para que explicaran si la habían llevado a cabo.

La secuencia de enseñanza-aprendizaje de la lectoescritura que tenían programada y que seguía el libro utilizado fue la siguiente. Se presentaba el grafema, tanto en minúscula como en mayúscula, con su sonido. Se asociaba a un dibujo, a un gesto y a un sonido. Posteriormente, se introducía el fonema con las distintas vocales, para luego trabajar palabras monosílabas y bisílabas, con sentido, que contenían el fonema. Oralmente, se trabajaban palabras que contenían el fonema al inicio y al final. Luego, a través de fichas, se trabajaba la grafía. A continuación, se incluían palabras combinadas con el fonema en cuestión y con otros fonemas anteriormente aprendidos. Se trabajaban estas palabras de forma global, buscando la comprensión. Finalmente, se elaboraban pequeñas frases con las palabras aprendidas.

\section{Procedimiento}

Una vez asignados los grupos al azar (grupo experimental y grupo control), todos los participantes fueron evaluados por profesionales de la educación (maestras especialistas en Audición y Lenguaje, psicopedagogas y psicólogas) previamente entrenados.

La evaluación inicial de los alumnos se llevó a cabo de forma individual en espacios asignados para ese fin y dentro del horario escolar. Cada alumno fue evaluado en velocidad de denominación y en conciencia fonológica utilizando la prueba para la evaluación del conocimiento fonológico PECO cuando cursaban $2 .^{\circ}$ y $3 .^{\circ}$ de infantil ( 4 y 5 años, respectivamente) y $1 .^{\circ}$ de primaria ( 6 años). A estos alumnos, se les aplicó el programa de intervención durante parte de ese curso y en los 2 cursos siguientes. Cuando los alumnos finalizaban $1 .^{\circ}, 2 .^{\circ}$ y $3 .^{\circ}$ de primaria, se evaluó la escritura, de forma grupal, utilizando parte o la totalidad del PROESC (Cuetos et al., 2002).

La intervención se llevó a cabo con el consentimiento de la inspección educativa, del equipo directivo de los colegios, de las

Tabla 4

Puntuaciones del grupo experimental y el grupo control en las tareas de denominación rápida

\begin{tabular}{|c|c|c|c|c|c|c|}
\hline Curso & Tarea & G. experimental Media (DT) & G. control Media (DT) & $\mathrm{gl}$ & $\mathrm{F}$ & $\mathrm{p}$ \\
\hline \multirow[t]{3}{*}{ Segundo de infantil } & Dibujos & $61,67(14,09)$ & $64,35(18,89)$ & 1,97 & 0,62 & 0,433 \\
\hline & Colores & $74,27(28,59)$ & $69,39(19,39)$ & & 1,01 & 0,317 \\
\hline & Letras & $62,82(34,98)$ & $52,15(42,31)$ & & 1,82 & 0,180 \\
\hline \multirow[t]{3}{*}{ Tercero de infantil } & Dibujos & $53,59(9,25)$ & $47,81(8,43)$ & 1,80 & 8,51 & 0,005 \\
\hline & Colores & $56,65(17,25)$ & $50,94(13,08)$ & & 2,71 & 0,103 \\
\hline & Letras & $38,30(15,50)$ & $43,36(14,15)$ & & 2,31 & 0,132 \\
\hline \multirow[t]{3}{*}{ Primero de primaria } & Dibujos & $47,17(9,83)$ & $46,51(14,95)$ & 1,88 & 0,06 & 0,804 \\
\hline & Colores & $46,96(13,48)$ & $46,53(17,99)$ & & 0,01 & 0,899 \\
\hline & Letras & $34,91(15,71)$ & $32,44(9,23)$ & & 1,09 & 0,298 \\
\hline
\end{tabular}


Tabla 5

Ejemplo de contenidos, actividades y materiales para el objetivo: segmentar palabras en sílabas

\begin{tabular}{|c|c|c|c|c|}
\hline Curso & Contenidos & Actividades & Materiales & Secuencia de enseñanza \\
\hline Segundo de infantil & $\begin{array}{l}\text { Segmentar frases en palabras, } \\
\text { identificar vocales, p, m, s, l, f, n, d, } \\
\text { identificar sílabas (iniciales y finales), } \\
\text { palabras que riman, mezclar sonidos, } \\
\text { leer y escribir palabras combinando } \\
\text { sonidos y sílabas } \\
\text { Para velocidad de denominación: } \\
\text { nombrar dibujos, colores y letras en un } \\
\text { panel con velcros }\end{array}$ & $\begin{array}{l}\text { 1. Se reparten las tarjetas con } \\
\text { imágenes. Cada niño colocará en el } \\
\text { panel, debajo del número } \\
\text { correspondiente, la tarjeta que tiene } \\
\text { ese número de sílabas } \\
\text { 2. El niño debe colocar en el recuadro } \\
\text { el número de sílabas correspondientes } \\
\text { a cada dibujo (cuadernillo del niño 3.2) } \\
\text { 3. Cada niño debe buscar, en el libro } \\
\text { mágico de las sílabas, al menos } 3 \\
\text { palabras que el profesor le indique }\end{array}$ & $\begin{array}{l}\text { 1. Tarjetas con } \\
\text { imágenes y panel } \\
\text { con velcros o tabla } \\
\text { en el encerado con } \\
4 / 5 \text { columnas } \\
\text { 2. Cuadernillo del } \\
\text { niño y lápiz } \\
\text { 3. Libro mágico de } \\
2 \text { sílabas }\end{array}$ & $\begin{array}{l}\text { Explicación, demostración, } \\
\text { práctica grupal y práctica } \\
\text { independiente para todas } \\
\text { las actividades de } \\
\text { conciencia fonológica } \\
\text { Para la velocidad de } \\
\text { denominación, en cada } \\
\text { sesión, por turnos, los } \\
\text { niños nombraban los } \\
\text { elementos de un panel y se } \\
\text { registraban los tiempos. } \\
\text { Los elementos del panel se } \\
\text { cambiaban cada semana }\end{array}$ \\
\hline Tercero de Infantil & $\begin{array}{l}\text { Segmentar palabras en sílabas; } \\
\text { identificar n, l, s, d, r, z, palabras que } \\
\text { riman; identificar sílabas medias; } \\
\text { mezclar sonidos, letras y sílabas; } \\
\text { escribir y leer seudopalabras } \\
\text { Para velocidad de denominación: } \\
\text { nombrar dibujos, colores y letras en un } \\
\text { panel con velcros }\end{array}$ & $\begin{array}{l}\text { 1. Se reparten las tarjetas con los } \\
\text { dibujos/palabras y cada niño debe } \\
\text { decir qué es (imágenes diferentes al } \\
\text { nivel anterior). Todos los demás deben } \\
\text { marcar con las palmas las sílabas, y el } \\
\text { niño meterá la tarjeta en la bolsa } \\
\text { correspondiente que indicará el } \\
\text { número de sílabas } \\
\text { 2. Se reparten las tarjetas. Cada niño } \\
\text { debe decir el nombre del dibujo, } \\
\text { cuántos trocitos tiene y pegar la tarjeta } \\
\text { debajo del número correspondiente en } \\
\text { el panel } \\
\text { 3. Cada niño debe buscar la sílaba que } \\
\text { tienen en común } 2 \text { dibujos y escribirla } \\
\text { en el recuadro correspondiente } \\
\text { (cuadernillo del niño } 3.3 \text { ) }\end{array}$ & $\begin{array}{l}\text { 1. Tarjetas con } \\
\text { dibujos y bolsas } \\
\text { con los números de } \\
2 \text { a } 5 \\
\text { 2. Panel con los } \\
\text { números } 2,3,4 \text { y } 5 \\
\text { y tarjetas de la } \\
\text { sesión } 1 \\
\text { 3. Cuadernillo del } \\
\text { niño y lápiz }\end{array}$ & \\
\hline Primero de Primaria & $\begin{array}{l}\text { Jugar con los sonidos iniciales f, c, l, p, } \\
\text { b, s, m, t; finales l, s, r, n, z; identificar } \\
\text { palabras que riman identificar sílabas } \\
\text { medias; mezclar sonidos, letras y } \\
\text { sílabas; escribir y leer seudopalabras } \\
\text { Para velocidad de denominación: } \\
\text { nombrar dibujos, colores y letras en un } \\
\text { panel con velcros }\end{array}$ & $\begin{array}{l}\text { 1. Se reparten las tarjetas con las } \\
\text { imágenes/palabras y cada niño debe } \\
\text { decir qué es (imágenes diferentes del } \\
\text { nivel anterior) } \\
\text { Todos los demás deben marcar con las } \\
\text { palmas las sílabas, y el niño meterá la } \\
\text { tarjeta en la bolsa correspondiente que } \\
\text { indicará el número de sílabas } \\
\text { 2. Se reparten las tarjetas y cada niño } \\
\text { debe decir el nombre del dibujo, } \\
\text { cuántas sílabas tiene, y colocarla } \\
\text { debajo del número correspondiente en } \\
\text { el panel de las sílabas } \\
\text { 3. Cada niño debe buscar la sílaba que } \\
\text { tienen en común } 2 \text { dibujos y escribirla } \\
\text { en el recuadro (cuadernillo del niño } \\
\text { 2.3) }\end{array}$ & $\begin{array}{l}\text { 1. Tarjetas con } \\
\text { dibujos y bolsas } \\
\text { con los números de } \\
2 \text { a } 5 \\
\text { 2. Panel con los } \\
\text { números } 2,3,4,5 \\
\text { 3. Cuadernillo del } \\
\text { niño y lápiz }\end{array}$ & \\
\hline
\end{tabular}

profesoras-tutoras y de los padres de los alumnos de los cursos participantes, siendo informados del procedimiento. El grupo experimental recibió instrucción explícita en conciencia fonológica y velocidad de denominación a través de un programa diseñado para tal fin. Los alumnos que iniciaron la instrucción en $2 .^{\circ}$ de infantil ( 4 años) tuvieron 18 sesiones de intervención el primer año, 36 sesiones el $2 .^{\circ}$ año (cuando cursaban $3 .^{\circ}$ de infantil) y 36 sesiones el tercer año (cuando estaban en $10^{\circ}$ de primaria). Los alumnos que empezaron la intervención en $3 .^{\circ}$ de infantil ( 5 años) tuvieron 18 sesiones en ese curso y 36 en el año siguiente cuando cursaban $1 .{ }^{\circ}$ de primaria. Los alumnos que empezaron la instrucción en $1 .^{\circ}$ de primaria ( 6 años) tuvieron 18 sesiones de intervención en ese curso. Por otra parte, los alumnos del grupo control no participaron en la fase de intervención, sino que recibieron la enseñanza reglada con los objetivos curriculares oficiales de Educación Infantil y Educación Primaria establecidos por el organismo competente. Eso significa que recibieron enseñanza sobre escritura pero no sobre conciencia fonológica ni velocidad de denominación.

Los profesores del grupo experimental recibieron entrenamiento durante 2 sesiones, en las que se les explicó el procedimiento. A cada uno se le entregaron los objetivos, las instrucciones, las actividades diseñadas para cada objetivo y para cada sesión, los materiales pertinentes, así como unas hojas de anotaciones para valorar la consecución de los objetivos que eran revisadas, semanalmente, por los investigadores para subsanar cualquier dificultad respecto a los objetivos, materiales, actividades, etc. Al finalizar cada curso, se hacía seguimiento a los cuadernillos de los alumnos, de tal manera que se pudo observar la evolución de cada alumno al terminar cada curso y al finalizar los 3 años del estudio.

Los alumnos del grupo control fueron evaluados con las mismas actividades (conciencia fonológica, velocidad de denominación y escritura) y en los mismos tiempos que el grupo experimental.

El grupo control continuó con el programa establecido en el currículum y no recibió ayuda adicional en tareas de lectoescritura.

Los dos grupos utilizaban, para el aprendizaje de la lectura y de la escritura, métodos comercializados que suponen primero, el aprendizaje de las vocales y posteriormente el de las consonantes para trabajar luego la formación de silabas.

\section{Resultados}

Para analizar la eficacia del programa, se realizó un análisis estadístico con el modelo ANCOVA donde la variable de interés o 
Tabla 6

Puntuaciones del grupo experimental y el grupo control en las tareas de conciencia fonológica después de la intervención

\begin{tabular}{|c|c|c|c|c|c|c|c|}
\hline Curso & Tarea & $\begin{array}{l}\text { G. exper. } \\
\text { Media (DT) }\end{array}$ & $\begin{array}{l}\text { G. control } \\
\text { Media (DT) }\end{array}$ & gl & $\mathrm{F}$ & $\mathrm{p}$ & Tamaño efecto. Eta cuadrado \\
\hline \multirow[t]{6}{*}{ Segundo de infantil } & Identificación de sílabas & $5,00(0,00)$ & $4,872(0,33)$ & 1,96 & 6,567 & 0,012 & 0,063 \\
\hline & Identificación de fonemas & $4,96(0,20)$ & $4,78(0,50)$ & 1,97 & 4,936 & 0,029 & 0,048 \\
\hline & Adición de sílabas & $4,31(0,87)$ & $3,83(1,19)$ & 1,97 & 4,980 & 0,028 & 0,049 \\
\hline & Adición de fonemas & $4,49(0,72)$ & $3,93(1,33)$ & 1,97 & 6,452 & 0,013 & 0,062 \\
\hline & Omisión de sílabas & $4,47(1,03)$ & $4,39(0,87)$ & 1,97 & 0,164 & 0,687 & 0,002 \\
\hline & Omisión de fonemas & $4,27(1,13)$ & $3,78(1,35)$ & 1,97 & 3,692 & 0,058 & 0,037 \\
\hline \multirow[t]{6}{*}{ Tercero de infantil } & Identificación de sílabas & $4,98(0,14)$ & $4,94(0,33)$ & 1,80 & 0,380 & 0,540 & 0,005 \\
\hline & Identificación de fonemas & $6,04(7,07)$ & $4,64(0,54)$ & 1,80 & 1,408 & 0,239 & 0,017 \\
\hline & Adición de sílabas & $4,76(0,48)$ & $4,36(0,96)$ & 1,80 & 6,050 & 0,016 & 0,070 \\
\hline & Adición de fonemas & $4,30(0,98)$ & $3,72(1,03)$ & 1,80 & 6,762 & 0,011 & 0,078 \\
\hline & Omisión de sílabas & $4,85(0,36)$ & $4,17(1,13)$ & 1,80 & 14,717 & 0,000 & 0,155 \\
\hline & Omisión de fonemas & $4,52(0,80)$ & $3,89(1,30)$ & 1,80 & 7,267 & 0,009 & 0,083 \\
\hline \multirow{6}{*}{ Primero de primaria } & Identificación de sílabas & $5,00(0,00)$ & $5,00(0,00)$ & 1,88 & $\mathrm{NC}$ & $\mathrm{NC}$ & $\mathrm{NC}$ \\
\hline & Identificación de fonemas & $4,89(0,31)$ & $4,91(0,29)$ & 1,88 & 0,044 & 0,835 & 0,000 \\
\hline & Adición de sílabas & $4,91(0,28)$ & $4,60(0,72)$ & 1,88 & 7,333 & 0,008 & 0,077 \\
\hline & Adición de fonemas & $4,60(0,74)$ & $4,33(1,01)$ & 1,88 & 2,098 & 0,151 & 0,023 \\
\hline & Omisión de sílabas & $4,98(0,14)$ & $4,51(0,63)$ & 1,88 & 24,327 & 0,000 & 0,217 \\
\hline & Omisión de fonemas & $4,70(0,54)$ & $4,07(1,20)$ & 1,88 & 10,590 & 0,002 & 0,107 \\
\hline
\end{tabular}

NC: equivale a no calculable.

Tabla 7

Puntuaciones del grupo experimental y el grupo control en las tareas de denominación rápida después de la intervención

\begin{tabular}{|c|c|c|c|c|c|c|c|}
\hline Curso & Tarea & G. exper.Media (DT) & G. controlMedia (DT) & $\mathrm{gl}$ & $\mathrm{F}$ & $\mathrm{P}$ & Tamaño del efectoEta cuadrado \\
\hline \multirow[t]{3}{*}{ Segundo de infantil } & Dibujos & $40,42(9,82)$ & $42,00(9,759)$ & 1,97 & 0,64 & 0,426 & 0,007 \\
\hline & Colores & $39,98(10,64)$ & $42,89(15,67)$ & 1,97 & 1,12 & 0,292 & 0,011 \\
\hline & Letras & $24,38(11,19)$ & $26,22(5,95)$ & 1,97 & 1,09 & 0,298 & 0,011 \\
\hline \multirow[t]{3}{*}{ Tercero de infantil } & Dibujos & $35,83(6,90)$ & $37,56(8,58)$ & 1,80 & 1,02 & 0,315 & 0,013 \\
\hline & Colores & $33,78(5,93)$ & $37,14(7,19)$ & 1,80 & 5,35 & 0,023 & 0,063 \\
\hline & Letras & $20,50(3,03)$ & $23,17(5,49)$ & 1,80 & 7,80 & 0,007 & 0,089 \\
\hline \multirow[t]{3}{*}{ Primero de primaria } & Dibujos & $32,04(5,34)$ & $33,12(6,95$ & 1,88 & 0,68 & 0,412 & 0,008 \\
\hline & Colores & $31,40(5,85)$ & $33,23(8,92)$ & 1,88 & 1,34 & 0,250 & 0,015 \\
\hline & Letras & $18,77(3,55)$ & $18,98(2,53)$ & 1,88 & 0,11 & 0,745 & 0,001 \\
\hline
\end{tabular}

respuesta es la puntuación del postest de cada uno de los aspectos estudiados, siendo el factor intrasujetos, el grupo (experimental y control) y la variable explicativa el resultado del pretest. Los resultados de estos análisis se muestran en las tablas 6-8 donde se presentan las puntuaciones medias de los grupos experimental y control en las tareas evaluadas, así como el tamaño de los efectos y los valores $\mathrm{F}$, grados de libertad y significación del contraste de influencia del factor grupo. Las tablas 6 y 7 muestran los resultados de las tareas de denominación rápida y conciencia fonológica, y en la tabla 8 se muestran los resultados para las tareas de escritura en cada uno de los cursos (1. ${ }^{\circ}$, $2 .^{\circ}$ y $3 .^{\circ}$ de primaria). Se debe tener en cuenta que la escritura se evaluó al terminar el estudio, en los cursos en que se iba consolidando el aprendizaje de la misma y, dado que no hay pretest, el modelo estadístico ajustado es un ANOVA con un factor (grupo).
En las tareas de conciencia fonológica se observa que el factor grupo influye en adición de fonemas en $2 .^{\circ}$ y $3 .^{\circ}$ de infantil pero no en $1 .^{\circ}$ de primaria; en todo caso, el tamaño del efecto es pequeño y en ningún caso explica más del 7,8\% de variabilidad. En omisión de sílabas, el programa no influye significativamente en $2 .^{\circ}$ de infantil pero sí influye significativamente en $3 .^{\circ}$ de infantil y $10^{\circ}$ de primaria, donde el tamaño del efecto está entre el 15,5 y el $21,7 \%$, respectivamente.

En omisión de fonemas, no es significativo al 0,05 en $2 .^{\circ}$ de infantil pero sí lo es en $3 .^{\circ}$ de infantil y $1 .^{\circ}$ de primaria. En este caso, el tamaño del efecto está entre el 8 y el $10 \%$.

En adición de sílabas, aunque el tamaño del efecto es pequeño (7\%), resulta significativo para los 3 cursos.

En identificación de sílabas de $1 .^{\circ}$ de primaria, no hay variabilidad ni influencia del factor. Todos los alumnos alcanzan la máxima puntuación.

Tabla 8

Puntuaciones del grupo experimental y el grupo control en las tareas de escritura

\begin{tabular}{|c|c|c|c|c|c|c|c|}
\hline Curso & Tarea & G. exper.Media (DT) & G. controlMedia (DT) & $\mathrm{gl}$ & $\mathrm{F}$ & $\mathrm{P}$ & $\begin{array}{l}\text { Tamaño del efecto } \\
\text { Eta cuadrado }\end{array}$ \\
\hline \multirow[t]{3}{*}{ Primero de primaria } & Dictado de sílabas & $23,56(2,05)$ & $21,59(2,48)$ & 1,97 & 17,91 & 0,000 & 0,156 \\
\hline & Dictado de seudopal. Total & $17,44(4,07)$ & $16,20(3,67)$ & 1,97 & 2,53 & 0,114 & 0,026 \\
\hline & Dictado de seudopal. regla ortog. & $8,69(2,58)$ & $6,57(3,73)$ & 1,57 & 5,74 & 0,020 & 0,092 \\
\hline \multirow[t]{5}{*}{ Segundo de primaria } & Dictado de sílabas & $24,28(1,08)$ & $22,89(1,50)$ & 1,80 & 23,61 & 0,000 & 0,228 \\
\hline & Dictado de palabras ortogra. arbit. & $14,70(3,17)$ & $14,00(4,50)$ & 1,80 & 0,67 & 0,415 & 0,008 \\
\hline & Dictado de palabras ortografía reglada & $18,93(2,95)$ & $16,64(4,14)$ & 1,80 & 8,55 & 0,004 & 0,097 \\
\hline & Dictado de seudopalabras total & $20,33(3,30)$ & $17,19(2,41)$ & 1,80 & 22,83 & 0,000 & 0,222 \\
\hline & Dictado de seudopalabras regla ortog. & $11,13(2,71)$ & $8,61(1,91)$ & 1,80 & 22,30 & 0,000 & 0,218 \\
\hline \multirow[t]{5}{*}{ Tercero de primaria } & Dictado de sílabas & $24,09(1,01)$ & $22,81(1,63)$ & 1,88 & 19,935 & 0,000 & 0,185 \\
\hline & Dictado de palabras ortografía arbit. & $17,89(3,48)$ & $17,07(3,88)$ & 1,88 & 1,12 & 0,292 & 0,013 \\
\hline & Dictado de palabras ortografía reglada & $19,94(3,71)$ & $19,74(3,25)$ & 1,88 & 0,07 & 0,796 & 0,001 \\
\hline & Dictado de seudopalabras total & $20,51(3,90)$ & $19,60(2,21)$ & 1,88 & 1,79 & 0,184 & 0,020 \\
\hline & Dictado de seudopalabras regla ortog. & $11,72(2,34)$ & $10,98(3,18)$ & 1,88 & 1,62 & 0,206 & 0,018 \\
\hline
\end{tabular}


En velocidad de denominación, en $3 .^{\circ}$ de infantil, el programa produce mejoras significativas en velocidad de denominación de colores y letras. Siendo el tamaño del efecto moderado, del 6 y el $9 \%$, respectivamente. También se observa que la media en todas las variables y cursos es menor en el grupo experimental que en el grupo control. Estas diferencias, siempre a favor del grupo experimental, no son grandes y, dado que el tamaño muestral tampoco lo es, las diferencias no son significativas.

En escritura, para el dictado de sílabas, el programa es claramente significativo en los 3 grupos y el tamaño del efecto está entre el 15 y el $22 \%$. Para el dictado de seudopalabras total en $2 .^{\circ}$ de primaria, el tamaño del efecto es del $22 \%$. Para dictado de seudopalabras con reglas ortográficas está entre el $9 \%$ para $1 .^{\circ}$ de primaria y el $21 \%$ en $2 .^{\circ}$ de primaria. En cambio, en $3 .^{\circ}$ de primaria, el grupo no es significativo y el tamaño del efecto es muy pequeño $(1,8 \%)$.

En 2 . $^{\circ}$ de primaria el grupo es significativo para dictado de palabras de ortografía reglada con un tamaño del efecto del 9,7\%. En este curso, el factor grupo es significativo en dictado de seudopalabras total y dictado de seudopalabras regladas. En este caso, el tamaño del efecto es del 22 el 21\%, respectivamente.

Tal como se puede observar en las tablas, se producen diferencias entre el grupo experimental y el control en 12 de las 16 tareas de conciencia fonológica debido a una mejoría en el grupo experimental, lo que indica que el programa de intervención fue efectivo. En denominación rápida solo hubo diferencias significativas en 2 , ambas en $3 .^{\circ}$ de infantil. Pero lo más importante son los resultados en la escritura, ya que en la mayoría de las tareas el grupo experimental obtuvo resultados estadísticamente mejores que el grupo control. En los 3 niveles se encontraron diferencias significativas entre el grupo experimental y el control en el dictado de sílabas. En 2. de primaria también se encontraron diferencias significativas en dictado de palabras de ortografía reglada, en el dictado de seudopalabras total y en el dictado de seudopalabras con reglas ortográficas. Y en $1 .^{\circ}$ de primaria en el dictado de seudopalabras con reglas ortográficas. Por lo tanto, el programa de intervención en denominación rápida conciencia fonológica mejoró los procesos básicos de escritura.

\section{Discusión}

El objetivo de este estudio era comprobar el efecto de la intervención en conciencia fonológica y velocidad de denominación sobre el aprendizaje de la escritura y los resultados obtenidos ponen de manifiesto que la instrucción en estas habilidades mejora algunos aspectos de la escritura, especialmente el dictado de sílabas, palabras y seudopalabras.

La puntuación en el dictado de sílabas es mejor en el grupo experimental de $1 .^{\circ}, 2 .^{\circ}$ y $3 .^{\circ}$ de primaria respecto al grupo control. Estos resultados se explicarían por la automatización de las reglas de conversión de fonema a grafema que permiten escribir de manera precisa cualquier combinación de letras para formar sílabas «regulares» y esto coincide con la hipótesis de que los niños, cuando aprenden a escribir, reconocen los fonemas de las palabras al pronunciarlas y que la conciencia fonológica se ve enriquecida por la escritura a través del conocimiento alfabético, es decir, por la aplicación de las reglas de conversión fonema-grafema, así como por las separaciones silábicas posibles en el texto escrito y por la escritura de sílabas complejas (Treiman, 1998; Mejía de Eslava y Eslava-Cobos, 2008).

Además, los alumnos de $2 .^{\circ}$ de primaria del grupo experimental obtuvieron mejores resultados en el dictado de palabras de ortografía reglada y en el total del dictado de seudopalabras. Se interpreta que la intervención en conciencia fonológica y en velocidad de denominación mejora el conocimiento ortográfico y la memoria de palabras específicas con regularidad ortográfica, dado que escribir una palabra facilita su representación léxica. Es decir, que las habilidades fonológicas preceden y son necesarias en los escritores principiantes para alcanzar las habilidades ortográficas. Ambas representaciones son intrínsecas a la representación de la palabra y la velocidad de denominación está asociada con el rápido procesamiento ortográfico (Sunseth y Bowers, 2002).

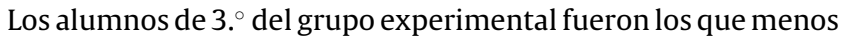
sesiones de instrucción recibieron (18) en conciencia fonológica y velocidad de denominación cuando estaban en $10^{\circ}$ de primaria. Esta puede ser una de las razones de encontrar pocas diferencias entre el grupo control y el experimental en las tareas de escritura. Otra posible interpretación de los resultados puede ser, como plantea Defior (2008), que al final del primer ciclo y cuando los niños alcanzan un nivel alto en la decodificación (aproximadamente en el período de 2 cursos escolares), termina la ayuda que supone la conciencia fonológica.

Los resultados obtenidos tienen implicaciones educativas relacionadas con el trabajo en el aula. Si los alumnos del grupo experimental obtuvieron mejores resultados que los del grupo control en algunas subpruebas de escritura, podemos inferir que la instrucción explícita en conciencia fonológica y velocidad de denominación mejora el aprendizaje de las escritura, al menos en los niveles básicos.

Las aportaciones más importantes que le hace la conciencia fonológica a la escritura se refieren a que proporciona herramientas para el deletreo temprano, favorece la comprensión lectora y desarrolla el reconocimiento de palabras, permitiendo, de manera eficaz, su segmentación en subunidades lingüísticas (Gillon, 2004). El alumno puede realizar, de manera temprana, correspondencias de fonema a grafema y viceversa, entender que estas conversiones son consistentes en la lengua española y así comprender el código alfabético.

A medida que los niños van siendo escritores más eficientes, dependen menos del procesamiento fonológico, ya que se consolida el procesamiento ortográfico que permite el acceso a la representación de las palabras de forma rápida, exacta y sin esfuerzo.

En este trabajo, también parece tener influencia la velocidad de denominación en el establecimiento y el uso de las representaciones ortográficas de palabras específicas. Además, facilita el acceso y la recuperación de los nombres de los símbolos visuales familiares que están almacenados en la memoria a largo plazo. Sin embargo, el papel de las capacidades de denominación parece menor, a juzgar por el menor número de tareas en las que los niños entrenados; sin embargo, fueron superiores a los del grupo control.

En resumen, este trabajo es relevante para la prevención de las dificultades en el aprendizaje de la escritura y para la identificación de los alumnos que están en riesgo de tener dificultades en la lectoescritura, especialmente a partir de tareas de conciencia fonológica. Permite orientar el diseño y el desarrollo de actividades educativas que incidan en las habilidades que se han identificado como relevantes en la adquisición y la automatización de procesos de bajo nivel. Asimismo, sería importante que en futuras investigaciones se considerara la influencia de estas variables en los procesos de alto nivel, como son la planificación, la organización y la revisión de la composición escrita.

Una limitación de este estudio es que el grupo control también ha estado realizando actividades lingüísticas relacionadas con la lectoescritura, pero a pesar de ello se han encontrado diferencias significativas entre el grupo experimental y el control en varias pruebas, lo que significa que la intervención realizada es efectiva.

A partir de los resultados de este estudio, parece recomendable diseñar programas de intervención ajustados a las necesidades de los alumnos que van a aprender a leer y escribir, con el fin de optimizar la automatización de los procesos básicos. Es importante diseñar programas de intervención que se puedan aplicar de manera integrada en el aula, teniendo en cuenta que la escritura es esencial para 
el éxito académico y la adaptación al medio social al garantizar la formación de «buenos escritores».

\section{Financiación}

Este artículo ha sido realizado dentro de un proyecto de investigación.

\section{Conflicto de intereses}

Los autores declaran no tener ningún conflicto de intereses.

\section{Referencias bibliográficas}

Alburquerque, C. (2012). Rapid naming contributions to reading and writing acquisition of European Portuguese. Reading and Writing, 25, 775-797.

Cuetos, F., Ramos, J. L. y Ruano, E. (2002). PROESC: batería de evaluación de los procesos de escritura. Madrid: TEA.

Caravolas, M. (2004). Spelling development in alphabetic writing systems: A crosslinguistic perspective. European Psychologist, 9(1), 3-14.

Defior, S. (2008). ¿Cómo facilitar el aprendizaje inicial de la lectoescritura? Papel de las habilidades fonológicas. Infancia y Aprendizaje, 31(3), 333-345.

Defior, S. y Serrano, F. (2011). Procesos fonológicos explícitos e implícitos, lectura y dislexia. Revista Neuropsicología, Neuropsiquiatría y Neurociencias, 11(1), 79-94.

De Jong, P. F. y van der Leij, A. (2002). Effects of phonological abilities and linguistic comprehension on the development of reading. Scientific Studies of Reading, 6, 51-77.

Furnes, B. y Samuelsson, S. (2011). Phonological awareness and rapid automatized naming predicting early development in reading and spelling: Results from a cross-linguistic longitudinal study. Learning and Individual Differences, 21, 85-95.

Gillon, G. T. (2004). Phonological awareness: From research to practice. New York: Guilford Press.

Guerrero, P. y López, A. (1992). Vocabulario básico para la educación infantil. Madrid: Bruño.

Hulme, Ch., Hatcher, P. J., Nation, N., Braun, A., Adams, J. y Stuart, G. (2002). Phoneme awareness is a better predictor of early reading skill than honest -rime awareness. Journal of Experimental Child Psychology, 82, 2-28.

Justicia, F. (1995). El desarrollo del vocabulario. Diccionario de frecuencias. Universidad de Granada.

Kessler, B. y Treiman, R. (2003). Is English spelling chaotic? Misconceptions concerning its irregularity. Reading Psychology, 24, 291-313.

Landerl, K. y Wimmer, H. (2008). Development of word reading fluency and spelling in a consistent orthography: An 8-year follow-up. Journal of Educational Psychology, 100, 150-161.

Manis, F. R., Seidenberg, M. S. y Doi, L. M. (1999). See Dick RAN: Rapid naming and the longitudinal prediction of reading subskills in first and second graders. Scientific Studies of Reading, 3(2), 129-157.

Manis, F. R., Doi, L. M. y Bhadha, B. (2000). Naming speed, phonological awareness, and orthographic knowledge in second graders. Journal of Learning Disabilities, 33, 325-333.

Manis, F. R. y Freedman, L. (2001). The relationship of naming speed to multiple reading reassures in disabled and normal readers. In M. Wolf (Ed.), Dyslexia, fluency, and the brain (pp. 65-92). Timonium, MD: York Press.
McCutchen, D. (2000). Knowledge, processing, and working memory: Implications for a theory of writing. Educational Psychologist, 35(1), 13-23.

Mejía de Eslava, L. y Eslava-Cobos, J. (2008). Conciencia fonológica y aprendizaje lector. Instituto Colombiano de Neurociencias. Acta Neurológica Colombiana, 24.

National Early Literacy Panel. (2008). Developing early literacy: Report of the Nationa Early Literacy Panel. Washington: National Institute for Literacy.

Onochie-Quintanilla, E., Simpson, I., Caravolas, M. y Defior, S. (2011). Letter knowledge, phoneme awareness and RAN as predictors of reading fluency in Spanish. In 10th Symposium of Psycholinguistics San Sebastián, April 13th-16th.

Parrila, R., Kirby, J. R. y McQuarrie, L. (2004). Articulation rate, naming speed, verba short-term memory, and phonological awareness: Longitudinal predictors of early reading development? Scientific Studies of Reading, 8, 3-26.

Ramos, J. L. y Cuadrado, I. (2006). Prueba para la evaluación del conocimiento fonológico. PECO. Madrid: EOS.

Romani, C., Olson, A. y di Betta, A. M. (2005). Spelling disorders. In M. J. Snowling y C. Hulme (Eds.), The science of reading: A handbook (pp. 431-448). Malden: Blackwell Publishing Ltd.

Savage, R., Pillay, V. y Melidona, S. (2008). Rapid serial naming is a unique predictor of spelling in children. Journal of Learning Disabilities, 41, 235-250.

Shanahan, T. (2004). Overcoming the dominance of communication: Writing to think and to learn. In T. L. Jetton y J. A. Dole (Eds.), Adolescent literacy research and practice. New York: Guilford Press.

Strattman, K. y Hodson, B. W. (2005). Variables that influence decoding and spelling in beginning readers. Child Language Teaching and Therapy, 21, 165-190.

Suárez-Coalla, P., García-de-Castro, M. y Cuetos, F. (2013). Variables predictoras de la lectura y la escritura en castellano. Infancia y Aprendizaje, 36(1), 77-89.

Sunseth, K. y Bowers, P. G. (2002). Rapid naming and phonemic awareness: Contributions to reading, spelling and orthographic knowledge. Scientific Studies of Reading, 6(4), 401-429.

Swanson, H. L. y Berninger, V. (1996). Invidual differences in children's working memory and writing skills. Journal of Experimental Child Psychology, 63 358-385.

Thomson, J.y Hogan, T. (2009). Future advances in the early detection of reading risk: Subgroups, dynamic relations, and advanced methodologies. Epilogue Special Edition "Advances in the Early Detection of Reading Risk". Journal of Learning Disabilities, 43(4), 383-386.

Treiman, R. (1998). Why spelling? The benefits of incorporating spelling into beginning reading instruction. In J. Metsala y L. Ehri (Eds.), Word recognition in beginning literacy (289-313). Mahwah, NJ: Lawrence Erlbaum.

Treiman, R. (2004). Phonology and spelling. In P. Bryant y T. Nunes (Eds.), Handbook of children's literacy (pp. 54-76). Dordrecht: Kluwer.

Verhagen, W., Aarnoutse, C. y van Leeuwe, J. (2010). Spelling and word recognition in Grades 1 and 2: Relations to phonological awareness and naming speed in Dutch children. Applied Psycholinguistics, 31, 59-80.

Wagner, R. K., Torgesen, J. K. y Rashotte, C. A. (1994). Development of reading-related phonological processing abilities: New evidence of bidirectional causality from a latent variable longitudinal study. Developmental Psychology, 30(1), 73-87.

Wolf, M. y Denckla, M. B. (2005). The rapid automatized naming and rapid alternating stimulus tests. Examiner's manual. Austin: Pro-Ed.

Wolf, M., OŔourke, A., Gidney, C., Lovett, M., Cirino, P. y Morris, R. (2002). The Second deficit: An investigation of the independence of phonological and naming speed deficits and developmental dyslexia. Reading and Writing, 15, 43-72.

Yuste, C. (2005a). Batería de aptitudes diferenciales y generales, BADyG-I. Madrid: CEPE.

Yuste, C. (2005b). Batería de aptitudes diferenciales y generales, BADyG-E1. Madrid: CEPE. 